\title{
Chemoselective Electrosynthesis Using Rapid Alternating Polarity
}

Authors: Yu Kawamata ${ }^{1 *}$, Kyohei Hayashi ${ }^{1}$, Ethan Carlson ${ }^{1}$, Shobin Shaji ${ }^{2}$, Dirk Waldmann ${ }^{2}$, Bryan J. Simmons ${ }^{3}$, Jacob Edwards ${ }^{3}$, Christoph W. Zapf ${ }^{3}$, Masato Saito ${ }^{1}$, Phil S. Baran ${ }^{1 *}$

\section{Affiliations:}

${ }^{1}$ Department of Chemistry, The Scripps Research Institute, 10550 North Torrey Pines Road, La Jolla, CA 92037, USA.

${ }^{2}$ IKA Works, Inc., 3550 General Atomics Court, MS G02/321, San Diego, CA 92121, USA.

${ }^{3}$ Bristol Myers Squibb, 10300 Campus Point Drive Suite 100, San Diego, CA 92121, USA.

*Correspondence to: yukawama@scripps.edu, pbaran@scripps.edu

\begin{abstract}
:
Challenges in the selective manipulation of functional groups (chemoselectivity) in organic synthesis have historically been overcome using either reagents/catalysts that tunably interact with a substrate or through modification to shield undesired sites of reactivity (protecting groups). Although electrochemistry offers precise redox control to achieve unique chemoselectivity, this approach often becomes challenging in the presence of multiple redox-active functionalities. Historically, electrosynthesis has been performed almost solely by using direct current (DC). In contrast, utilization of alternating current (AC) has been considered as an option to improve reaction efficiency rather than a way to achieve distinctly different reaction outcomes. Here we show how a unique type of waveform employed to deliver electric current - rapid alternating polarity (rAP) - enables control over reaction outcomes in the chemoselective reduction of carbonyl compounds, one of the most widely used reaction manifolds. The reactivity observed cannot be recapitulated using DC electrolysis or chemical reagents. The synthetic value brought by this new method for controlling chemoselectivity is vividly demonstrated in the context of classical reactivity problems such as chiral auxiliary removal and cutting-edge medicinal chemistry topics such as the synthesis of PROTACs.
\end{abstract}

\section{[Introduction]}

Controlling chemoselectivity in organic synthesis is widely regarded as one of the paramount challenges of the field. ${ }^{1}$ Indeed, tuning reactions in complex settings to obtain precise positionselective outcomes is often the key bottleneck in executing synthetic pathways. ${ }^{2}$ Although computational chemistry techniques can be applied in certain contexts, identifying the right reagent or conditions to achieve such selectivity is, in most cases, an empirical endeavor. For this reason, the toolbox of organic chemistry is replete with multiple ways to achieve the same outcome, and protecting groups are still widely employed to block undesired reactivity. ${ }^{3,4}$ Amongst various ways to control a reaction outcome, precise redox control based on electron transfer is an emerging trend addressed by employing photoinduced electron transfer or direct electrochemical techniques. Although organic electrosynthesis is gaining popularity due to its ability to enable specific redox reactions to take place by adjusting the potential applied to a reaction, ${ }^{5}$ the presence of both oxidation and reduction can complicate reaction outcomes when using a simple undivided cell. 
Thus far, little attention has been paid to the effect of electron flow on the selectivity of electrochemical transformations on organic molecules. To date, nearly all synthetically useful electrosynthetic techniques proceed via direct current (DC) - where the polarity of electrodes remains unchanged such that the flow of electrons is uni-directional (Figure 1A, left). ${ }^{5}$ This current will drive the oxidation and reduction of molecules on anodes and cathodes, respectively, with the reaction outcome being rationalized based on redox potential: a thermodynamic parameter used to describe the tendency of a molecule to accept or give electrons. ${ }^{6}$ Another form of electric current is alternating current (AC, see Supplementary Materials for the detailed classification $)^{7}-$ wherein electrons flow back and forth between two electrodes in a reaction, most often with a sinusoidal waveform. In contrast to DC, reaction control using AC has been far less explored in synthetic electrochemistry (Figure 1A, right). ${ }^{8}$ In fact, AC has only been studied as an engineering option that could potentially improve reaction efficiency when employed instead of DC in known reactions ${ }^{9-13}$; although intriguing applications has been emerging, the discovery of unique reaction outcomes through AC has yet to materialize. An additional layer of difficulty stems from the lack of readily available instrumentation to generate a suitable $\mathrm{AC}$ for evaluation by synthetic organic chemists. ${ }^{14}$ Herein we disclose a practical implementation of a unique form of AC electrolysis that uses a square waveform, rapid alternating polarity (rAP), which enables precise control of specific reaction pathways and remarkable chemoselectivity in synthetically relevant reduction processes for which no known direct chemical alternative currently exists.

rAP is a type of AC featuring a square waveform (Figure 1B), achieved by alternating the polarity of an electrode in the millisecond timescale while maintaining either the current or potential constant. The underlying software to access such a waveform was recently implemented in ElectraSyn 2.0, a widely employed commercial potentiostat, to facilitate widespread use of this method without any engineering barrier. While several intriguing applications of square waveform has been reported ${ }^{15,16}$, rAP was developed based on the following hypothesis: if the rate of polarity switching is faster than the rate of certain redox reactions on an electrode, a slower subset of electrochemical processes might be suppressed (Figure 1C). ${ }^{17,18}$ In other words, rAP would enable differentiation of redox reactions based on their relative reaction rate so as to enable chemoselective electrosynthesis. Such a method could bring a new dimension to reaction control where differentiation of multiple redox reactions and redox-active functionality are inherently challenging based only on redox potentials. An aspirational goal would therefore be to employ rAP in a functional-group-rich setting such as tetrapeptide 1, containing as many as 8 redox-active sites. To the best of our knowledge, fine-tuning site-specificity in a redox reaction by simply modulating the waveform of electric current applied is without precedent in the realm of organic synthesis. 


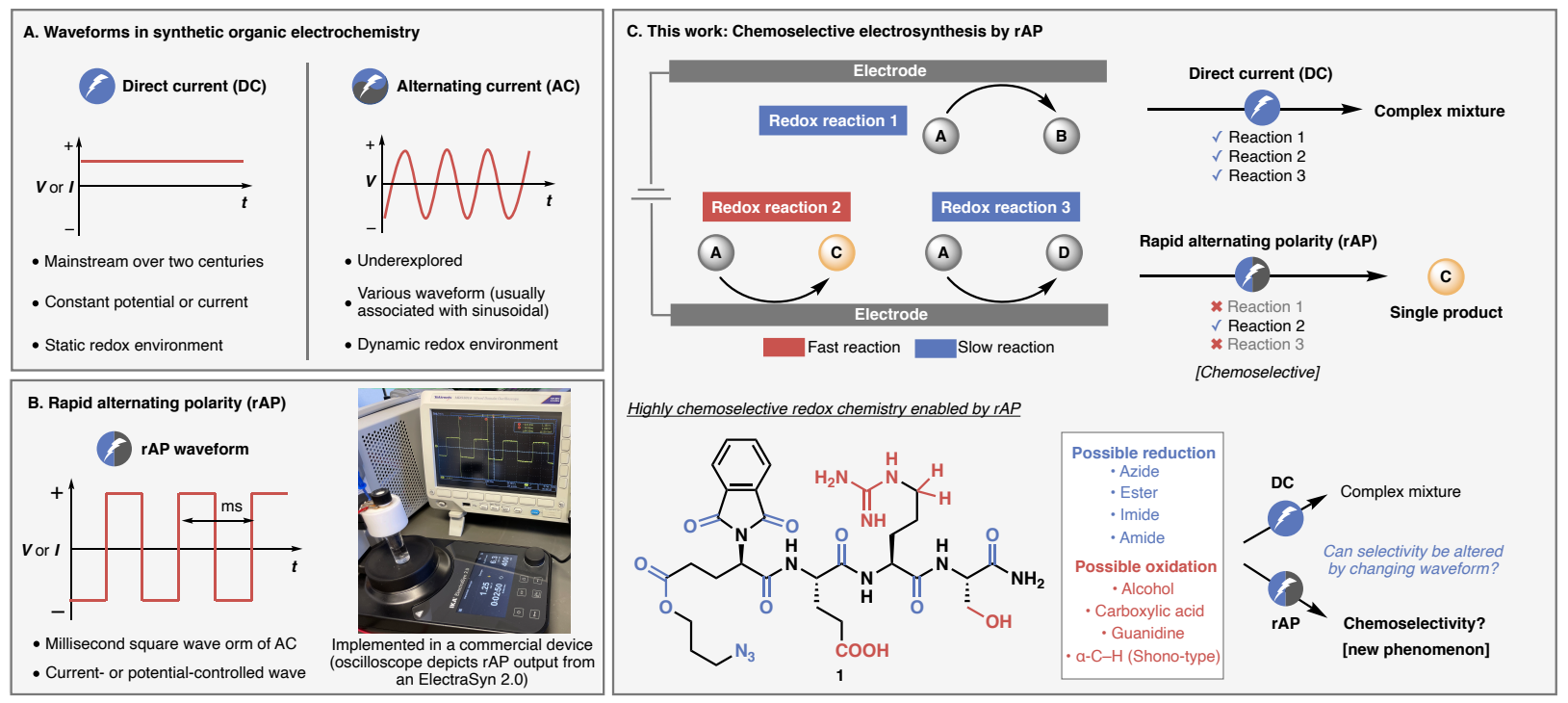

Figure 1. Types of waveforms in electrosynthesis and proposed reaction control enabled by rapid alternating polarity (rAP). (A) Illustration of direct current (DC) and alternating current (AC). (B) rAP waveform and implementation into a commercial device. (C) Hypothesis of achieving chemoselectivity by rAP through differentiating slow and fast electrochemical processes, and demonstration of its utility in a highly complex setting.

\section{[Proof of concept and reaction generality]}

To obtain proof-of-concept for such a hypothesis, piperidine derivative 2 (Figure 2A) was selected as a simple model substrate containing both oxidizable and reducible functional groups. Specifically, this structure contains both imide carbonyl groups and activated $\mathrm{C}-\mathrm{H}$ bonds, potentially susceptible to both reductive and oxidative functionalization, respectively. Figure 2 presents a detailed comparison of rAP with DC, as well as the influence of pulse duration (frequency) and waveform on the electrolysis of $\mathbf{2}$. Reaction conditions were chosen such that they could potentially lead to either reductive or oxidative outcomes (non-sacrificial electrodes, slightly acidic conditions). Each reaction was performed under identical conditions using pivalic acid as a proton source with electric current waveform (type/frequency/amplitude) being the only variable screened. Crude ${ }^{1} \mathrm{H}$ NMR analysis of the product distribution under standard DC electrolysis of phthalimide $\mathbf{2}$ revealed methoxy group incorporation into a large number of species resulting in a complex mixture (entry 1). Presumably, $\mathrm{C}-\mathrm{H}$ oxidation (Shono oxidation) occurred along with varying degrees of imide reduction leading to this unselective and synthetically unworkable outcome. ${ }^{19}$ To exclude the possibility that this unselective reaction profile was attributed to harsh electrolysis conditions, the current and amount of electrons were reduced (entries 2 and 3 ) to no avail. In stark contrast, rAP led to highly selective and efficient formation of either $\mathbf{3 a}$ or $\mathbf{3 b}$, depending on the pulse duration applied (constant current rAP, entries 4-8). Remarkably, methoxy group incorporation indicative of Shono oxidation was barely observable in these entries (except entry 4, slow rAP at $200 \mathrm{~ms}$ ). The crude ${ }^{1} \mathrm{H}$ NMR depictions of entries 1 and 6 vividly illustrate the stark difference in product distribution. At shorter pulse duration (25-33 ms, 15-20 Hz), the major product was hemiaminal 3a, whereas fully reduced lactam $\mathbf{3 b}$ was formed at longer pulse duration $(50-200 \mathrm{~ms}, 2.5-10 \mathrm{~Hz})$. This product distribution suggested that current efficiency is proportional to reduction strength and is a function of pulse duration: the longer the pulse is, the more redox reactions proceed (with an infinite pulse duration being equal to DC). The reduced current efficiency with a short pulse can be augmented by increasing the current, leading to a 
complete switch of product distribution from 3a to $\mathbf{3 b}$ [same $25 \mathrm{~ms}$ pulse but with 20 or $40 \mathrm{~mA}$, respectively (entry 8 vs entry 9)]. The same principle could be applied to achieve a selectivity switch in the opposite direction - from 3b to 3a [same $100 \mathrm{~ms}$ pulse but with 20 or $10 \mathrm{~mA}$, respectively (entry 5 vs entry 10)]. The highest yield of $\mathbf{3 b}$ was observed by adjusting the number of electrons $(16 \mathrm{~F} / \mathrm{mol})$ at $20 \mathrm{~mA}$ and $20 \mathrm{~ms}$ pulse duration (entry 11 ), resulting in $76 \%$ isolated yield after $1.5 \mathrm{~h}$ of electrolysis. The aforementioned rAP experiments were all run using constant current, but the technique could also be applied in a constant potential mode. Thus, potentialcontrolled rAP at a similar cell voltage to entry 11 (alternating $\pm 8 \mathrm{~V}$ ) with the same pulse duration also led to efficient formation of $\mathbf{3 b}$ (entry 12). On the other hand, the use of a sinusoidal waveform (conventional AC) at the same terminal potential and frequency gave 3a instead of $\mathbf{3 b}$, indicating a much lower current efficiency with this waveform (entry 13). ${ }^{10}$

Having established rAP as a unique mode of electrolysis distinctly different from conventional DC electrolysis or even rarely employed sinusoidal AC under identical reaction conditions, one question remained: is the observed reduction unachievable by DC with further reaction engineering? In principle, Shono oxidation products could be suppressed simply using a divided cell or employing a sacrificial anode to avoid such an oxidative side reaction. Towards that end, the reaction was carried out in a divided cell, furnishing 3a in low yield (entry 14). This inefficient reduction was partly accounted for the high resistance present in a divided cell setup. The reaction with a Zn sacrificial anode (entry 15) also resulted in a similar outcome (lactam $\mathbf{3 b}$ was not observed). Finally, employing a sacrificial electron donor $\left(\mathrm{e}^{-}\right.$-donor $\left.=i \operatorname{Pr}_{2} \mathrm{NH}\right)$ instead of a sacrificial anode, under the conditions reported by Xiang et al., indeed afforded a 2:1 mixture of 3a and $\mathbf{3 b}$ (entry 16). ${ }^{20}$ As presented below, further comparison with these conditions unveiled that rAP-based reduction exhibits a dramatically expanded scope and generality in both simple and demanding settings.

To further gauge the synthetic utility of reductive rAP over conventional DC electrolysis, it was field-tested in synthetically relevant contexts (Figure 2B). Isoindolinone derivatives are an important class of molecules in both medicinal and agricultural chemistry, and routes to such molecules usually employ stepwise ring construction rather than late-stage reduction of a phthalimide. ${ }^{21}$ Reductively labile alkene (4), alkyne (5), epoxide (6), alkyl bromide (7), and ketone (8) motifs were all tolerated in addition to the oxidatively labile $\mathrm{C}-\mathrm{B}$ bond (9). Efficient reduction of $N$-unsubstituted phthalimide $(\$ 0.1 / \mathrm{g})$ afforded a valuable building block $10(\$ 576 / \mathrm{g}$ in SigmaAldrich). In the case of heteroaromatic imides, hemiaminal was obtained as a sole product (1113). $N$-Ethoxycarbonyl phthalimide was found to be reduced cleanly to the hemiaminal 14 by employing $\mathrm{MeCN}$ as solvent. This reaction was also effective for a peptidic substrate bearing an unprotected alcohol, affording 15 in high yield. The synthetic utility was further demonstrated in the successful reduction of unprotected thalidomide on gram-scale, affording deoxythalidomide $\mathbf{1 6}^{22}$ in one step after simple recrystallization from the crude mixture. Amongst the various substrates studied, one of the most surprising selectivities was observed with phthalimide 17: selective deoxygenation of phthalimide without reducing the alkyl aldehyde (Figure 2C). This striking chemoselectivity was indeed unique to rAP as conventional direct electrolysis, both under the same reaction conditions as well as with $\mathrm{e}^{-}$-donor, led to extensive decomposition of 17. Wellestablished chemical reductants such as DIBAL and $\mathrm{LiBH}_{4}$ gave reduction of aldehyde preferentially and reported chemical conditions for phthalimide reduction gave a complex mixture. ${ }^{23}$ Finally, Figure 2D illustrates the generality of the rAP reduction across various types 
of carbonyl compounds. In general, electron-deficient carbonyl compounds are susceptible to this reduction, whereas simple esters, ketones, and amides are not affected (Figure 3A). Hemiaminals were cleanly formed in the reduction of various alkyl imides, including 22 from bicyclic succinimide derivative conveniently accessible by $\mathrm{Yu}$ 's $\mathrm{Pd}$-catalyzed $\mathrm{C}-\mathrm{H}$ functionalization. ${ }^{24}$ The reduction of $\mathrm{N}$-alkylhydantoins led to the formation of imidazolone instead of hemiaminal due to facile dehydration of the product hemiaminal. Although chemical reduction of fully protected hydantoins is known, reduction of mono-protected systems (to yield compounds such as 24) is challenging due to the free $\mathrm{N}-\mathrm{H} .{ }^{25}$ While the alkyl aldehyde was tolerated during the reduction of 17, hydrocinnamyl aldehyde 25 could be reduced under slightly modified rAP conditions by employing a longer pulse duration (see Supplementary Materials for detail). Exposure of the simple $N$-acylsulfonamide (27) to rAP led to a mixture of desulfonylated amide 8a and sulfonamide $\mathbf{8 b}$ (along with benzyl alcohol in 21\%). $\alpha, \beta$-Unsaturated ester 29 and thioester 31 could also be reduced to the corresponding saturated ester (30) and alcohol (32), respectively. Reduction of trifluoroacetamide-containing piperidine (33) delivered unexpected mono- and bisdefluorinated products $(\mathbf{3 4 a} / \mathbf{b})$ - such an outcome had no known chemical counterpart until the very recent discovery of radical-based defluorinative transformation, indicating that rAP could exhibit such interesting reactivity under simple conditions. ${ }^{26}$ Through the entirety of Figure 2 , conventional DC electrolysis with a sacrificial $\mathrm{e}^{-}$-donor was run in parallel with rAP-based reduction; in nearly all cases rAP was found to be superior with more complex cases failing completely under DC $(7,12,13,14,16,17,24,27,31)$. 


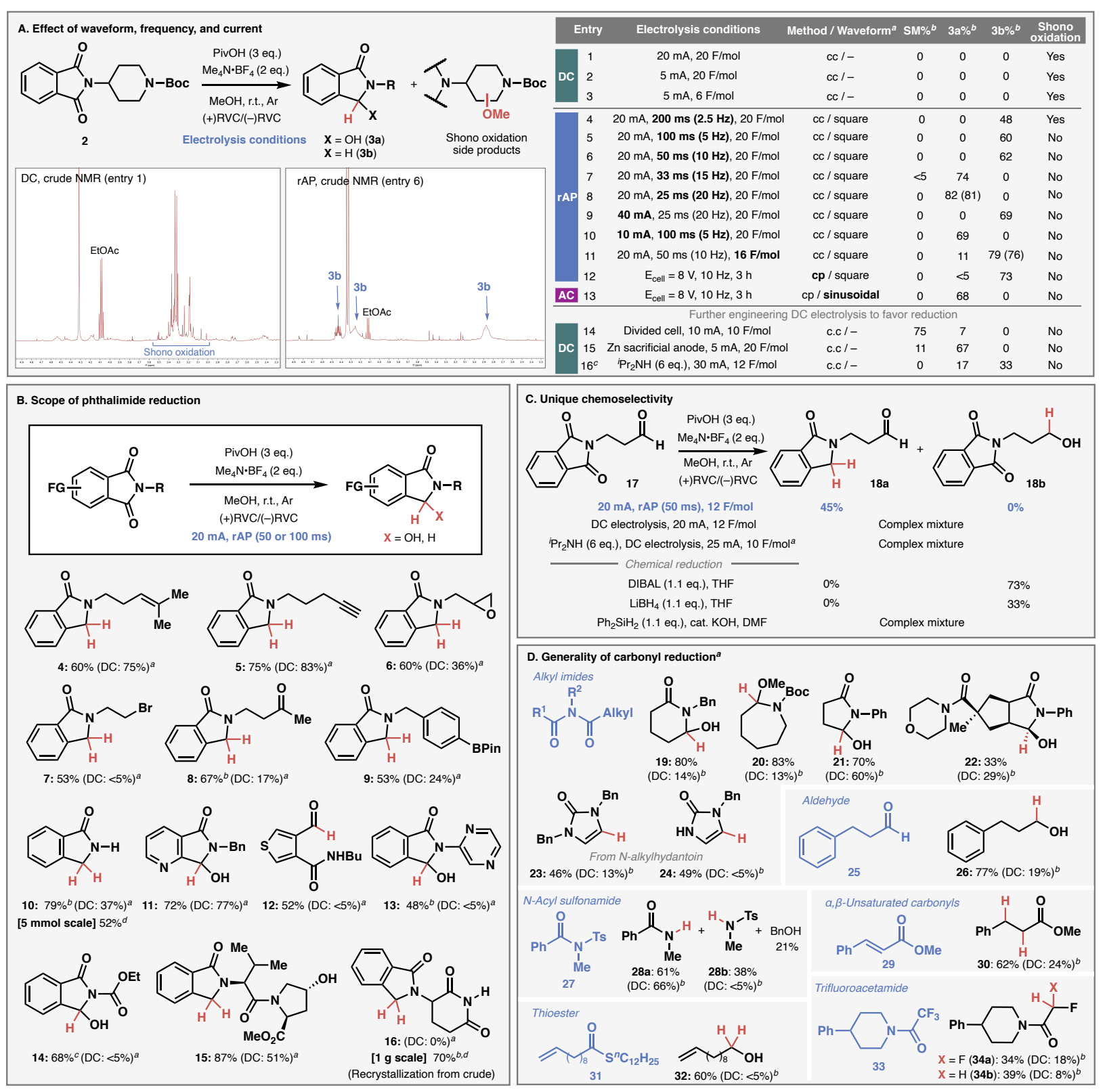

Figure 2.Difference between rAP and canonical DC electrolysis in general carbonyl reduction. (A) A striking difference in reaction outcome by employing $\mathrm{rAP}$ with various frequencies and currents. ${ }^{\mathrm{a}} \mathrm{cc}=$ constant $\mathrm{current}, \mathrm{cp}=$ constant potential. ${ }^{b} \mathrm{NMR}$ yield. Isolated yields are shown in parenthesis. ${ }^{\mathrm{c}}$ Conditions adopted from reference 20. (B) Functional group tolerance in the reduction of phthalimides benchmarked with the latest DC electrolysis conditions.

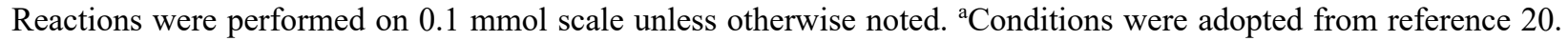
${ }^{b} \mathrm{MeCN} / \mathrm{tBuOH}$ (1:1) was used as solvent. ${ }^{\mathrm{c}} \mathrm{MeCN}$ was used as solvent. ${ }^{\mathrm{d}} 100 \mathrm{~mA}$ was applied instead of $20 \mathrm{~mA}$. (C) Discovery of chemoselectivity unachievable by known synthetic methods. ${ }^{\mathrm{a} C o n d i t i o n s}$ were adopted from reference 20. (D) Generality of carbonyl reduction by rAP. ${ }^{a}$ See SI for reaction conditions for each substrate. ${ }^{b} \mathrm{DC}$ conditions were adopted from reference 20.

\section{[Applications]}

The synthetic utility of the unique reactivity enabled by rAP was demonstrated in two popular contexts: chiral auxiliary removal and an emerging area of medicinal chemistry known as proteolysis targeting chimeras (PROTACs). In the first instance (Figure 3A), it is well-recognized 
that reductive removal of Evans auxiliary to provide an aldehyde is challenging especially in the presence of other reducible carbonyl groups. A recently reported synthesis of chiral aldehyde 37 is an emblematic example of this lingering issue. ${ }^{27}$ In order to prepare this simple aldehyde, a ninestep route involving three protecting group manipulations and five redox manipulations was required. Only one step forged a key $\mathrm{C}-\mathrm{C}$ bond (allylation) that needed several corrective concession steps to install the requisite $t$-butyl ester group. In contrast, commencing with the same starting material (35), direct alkylation with $t$-butyl acrylate followed by subjecting 36 to reductive rAP cleanly delivered the hemiaminal that could be easily cleaved during workup to reveal aldehyde 37. It was confirmed that other representative chemical reductants such as DIBAL and $\mathrm{LiBH}_{4}$ led to either reduction of the ester or overreduction of the desired aldehyde (see Supplementary Materials). Comparable DC electrolysis with or without an $\mathrm{e}^{-}$-donor led to a complex mixture of products (37 not detected). It is worth pointing out that the alkylation of acrylates with Evans auxiliary ${ }^{28}$ is a rarely employed tactic in synthesis, perhaps due to the heretofore intractable challenge of chemoselective reduction.

In the second major application of reductive rAP, molecules relevant to the burgeoning field of PROTACs were investigated. Pomalidomide- (bearing a phthalimide) and lenalidomide-type (bearing an isoindolinone) scaffolds are cereblon E3 ligase binders used as standalone treatments in oncology (Pomalyst ${ }^{\circledR}$ or Revlimid $\AA$ ) or are incorporated into PROTAC design to elicit protein degradation. The bioactivity of PROTAC structures can vary based on the presence or absence of a single carbonyl group. ${ }^{29}$ Historically such entities are prepared through a de novo synthesis, whereas late-stage single-step reductive deoxygenations are, to our knowledge, without precedent. As shown in Figure 3B, reductive rAP deoxygenation of PROTAC building block $\mathbf{3 8}$ smoothly proceeded in the presence of azide functionality with no protection on the glutarimide subunit. ${ }^{30}$ The complete PROTAC molecule, pomalidomide-JQ1 conjugate $\mathbf{4 0}^{31}$ was subjected to several reduction conditions, with rAP as the only method capable of reducing $\mathbf{4 0}$ in synthetically meaningful yield. Due to the sensitivity of the JQ1-heterocycle, a two-step tandem reduction was performed in this case.

The synthetic feasibility of this methodology was further explored by enlisting a small library of thalidomide-type analogs (Figure 3C). Under standard conditions, facile reduction was achieved to give the corresponding isoindolinone products in synthetically useful yield. Electron-donating

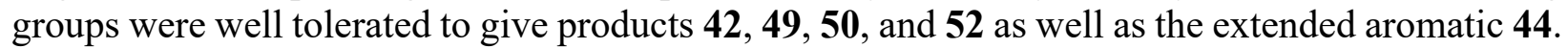
As suggested by products $\mathbf{4 3}, \mathbf{4 5}, \mathbf{4 6}$, and $\mathbf{5 1}$, electron-withdrawing groups were also able to withstand the reduction with dehalogenation being largely suppressed by the addition of TFA (for details, please see Supplementary Materials). The exquisite chemoselectivity of this transformation is highlighted by the incorporation of acyl and urea-protected amines (products 47 and 48). In both cases, no overreduction was observed with the desired products isolated in 23 and $48 \%$ yield, respectively.

With newly synthesized analogs in hand, they were analyzed in an HTRF-Tb (Homogeneous Time-Resolved Fluorescence - Terbium-based) ${ }^{32}$ cereblon-binding assay (Figure 3C, binding assay table). From this study, 9 out of 12 newly synthesized lenalidomide-type analogs showed greater binding affinity to cereblon than their thalidomide-type starting materials. Of note, 43 now shows sub-micromolar binding affinity, a drastic improvement to its parent compound having no measurable binding. Only in the cases of 48, 50, and 52a was a slight loss in affinity for cereblon 
observed. This exciting result underscores the benefit this new methodology may bring to the cereblon biochemistry field and, by extension, the protein degradation realm.
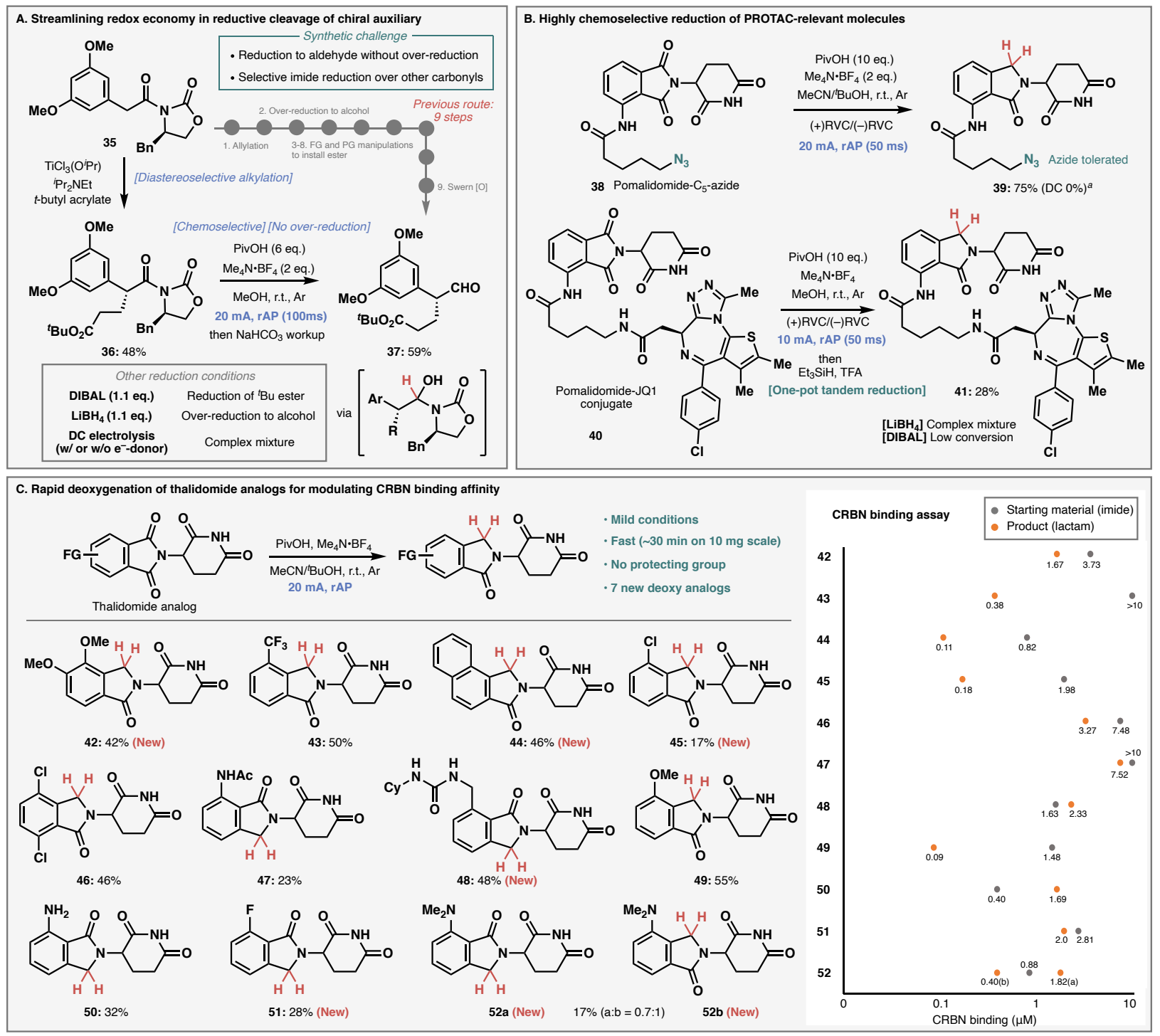

Figure 3. Synthetic applications of rAP. (A) Reductive removal of Evans auxiliary with high chemoselectivity among multiple carbonyl groups. (B) Highly chemoselective reduction for diversification of PROTAC molecules. (C) Rapid deoxygenation of thalidomide analogs and modulation of cereblon binding affinity.

\section{[Mechanistic investigations]}

The unconventional reduction outcomes observed on complex structures when employing rAP can be rationalized by redox potentials of organic functional groups as well as frontier molecular orbital theory (Figure 4A). From the standpoint of reduction potential, the phthalimide carbonyl has the highest reduction potential (ca. $-1.4 \mathrm{~V})$, followed by alkyl aldehyde (ca. $-1.8 \mathrm{~V}$ ), alkyl ketone (ca. $-2.4 \mathrm{~V}$ ), and amide/ester functionalities (both $<-2.5 \mathrm{~V}$ ). ${ }^{6,33}$ Thus, these reduction potentials clearly explain the rAP-based selectivity on substrates with multiple carbonyl groups such as $\mathbf{1 7}$ and 53. The difficulty in harnessing this difference using conventional chemical reductants could partly stem from the fundamental mechanistic difference in the reduction: with 
chemical reagents, amides and esters are generally reduced through the interaction between a carbonyl oxygen and a Lewis-acidic metal in a reagent. ${ }^{34}$ Even aldehyde, one of the most trivial functional groups to reduce by chemical reagents, was tolerated in the rAP-based reduction of 17, representing the most pronounced difference between rAP and chemical reductants. Simply stated, such selectivity is counter to textbook chemical intuition. Since the reduction potential corresponds to the LUMO (lowest unoccupied molecular orbital) based on Koopmans' theorem, the aforementioned selectivity can be rationalized based on the calculated LUMO coefficient, which provides convenient prediction even without relying on experimental electrochemical techniques such as cyclic voltammetry. As illustrated in Figure 4A, carbonyl carbons with the largest LUMO coefficient are in good agreement with experimental reactive sites (17 and 53). When there were several carbonyl carbons with similar LUMO coefficients, mixtures of products were obtained (54, vide infra). Thus, the selectivity observed invokes a single-electron transfer (SET)-based reduction to the radical anion, followed by protonation. Experimental support for this notion was obtained using substrate 54, wherein substantial dechlorination was observed under standard conditions, indicating the intermediacy of a radical anion (Figure 4B). ${ }^{35}$ Suppression of the dechlorination by the addition of strong acid such as TFA also indicates that the intermediate is sensitive to reaction $\mathrm{pH}$, supporting the existence of an anionic intermediate. The source of hydrogen could also be elucidated using substrate 56 under rAP in $\mathrm{MeOH}-d_{4}$ solvent wherein deuterium incorporation was observed on the methylene of lactam 58-d (Figure 4C). Such an inexpensive way of installing a stable-labeled isotope may find use in the synthesis of isotopically labeled materials. The fate of the carboxylic acid could also be followed in this reaction, delivering ether 59, derived from decarboxylative carbocation formation. ${ }^{36}$

The most striking feature of rAP is that it achieves selectivity control simply by virtue of its differing waveform, a phenomenon that has not been observed before. The unique parameter associated with rAP, namely frequency (pulse duration), was therefore studied in more depth. The frequency dependence of each elementary reaction - imide reduction, decarboxylation, and Shono oxidation - was interrogated individually (Figure 4D). Since the Shono oxidation is a competing reaction in phthalimide reduction to generate multiple products, simplified substrate $\mathbf{6 0}$ was used to obtain the frequency-dependent profile. ${ }^{37}$ Frequency had a major impact on the progress of the Shono oxidation; the yield of $\mathbf{6 1}$ was significantly reduced even at a frequency as low as $1 \mathrm{~Hz}(500$ ms pulse). The slow kinetics of the Shono oxidation are presumably related to intrinsically high overpotential associated with amide oxidation. ${ }^{38}$ Substrate $\mathbf{2}$ could be studied in a similar fashion with the converse observation that the yield of lactam $\mathbf{3 b}$ increased as frequency increased. The frequency had a relatively minor impact on the progress of oxidative decarboxylation of 57, although the yield of $\mathbf{5 9}$ decreases moderately as frequency increases. Taken together, a proposed mechanism consistent with these observations is depicted in Figure 4E. During the cathodic phase, reduction of the imide proceeds via SET followed by protonation. During the anodic phase of the pulse, the carboxylic acid is oxidatively decarboxylated, generating carbocation that is eventually trapped by solvent. Progress of the competitive Shono oxidation is considerably abrogated above a certain frequency, which accounts for reaction differentiation between $\mathrm{rAP}$ and conventional DC. Finally, to return to the peptidic substrate 1 mentioned at the outset of this work (Figure 1), exposure to rAP-based reduction resulted in 52\% NMR yield of lactam 62 without disrupting the numerous redox-active and epimerizable stereocenters. Such control of chemoselectivity represents a unique direction for synthetic organic electrochemistry and a practical technique that 
is currently outside the reach of conventional chemical reagent space. As such, it warrants further study from both mechanistic and reaction discovery standpoints.

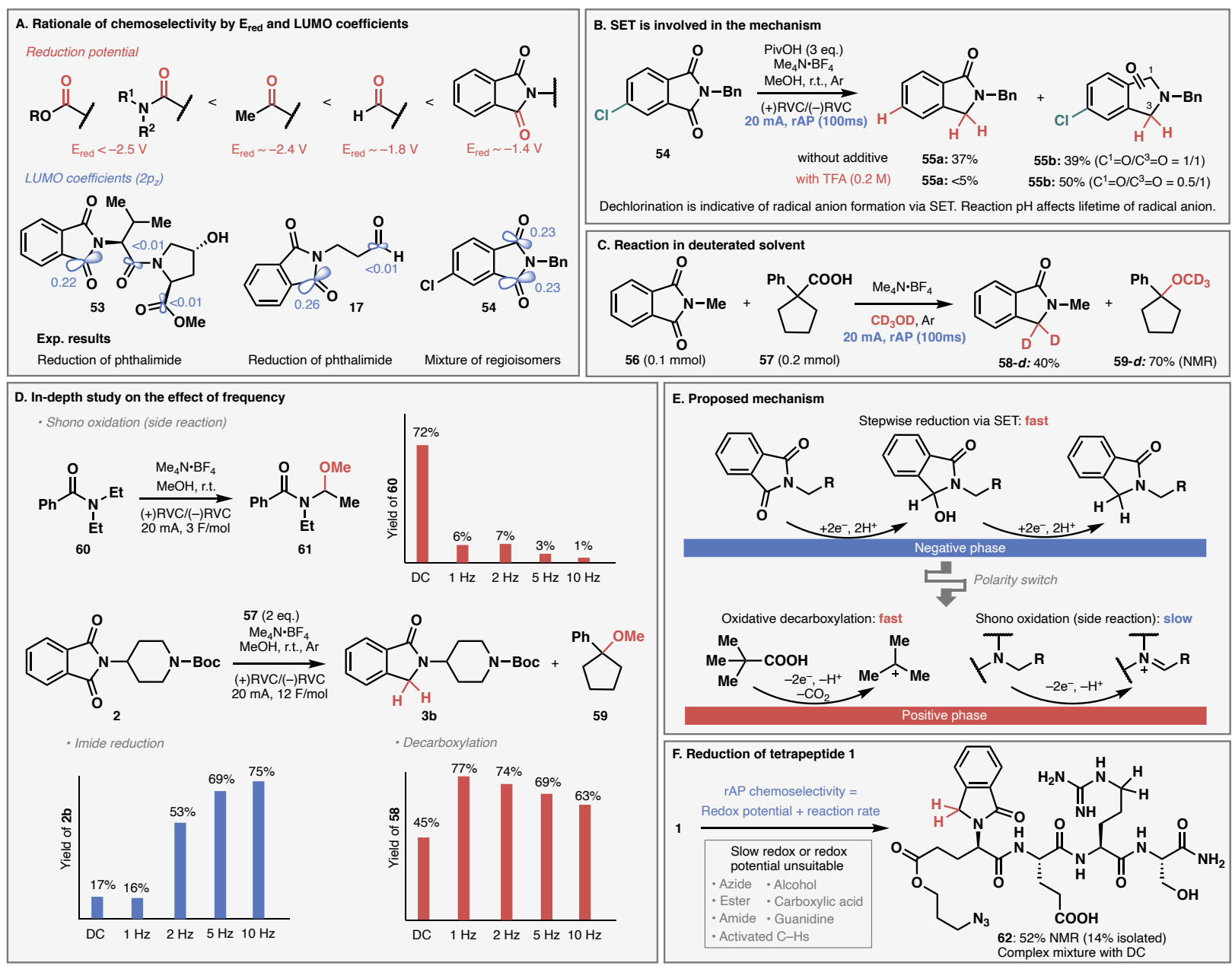

Figure 4. Experimental and computational rationale for unique reactivity of rAP. (A) Chemoselectivity follows reduction potential and can be predicted by FMO analysis. (B) Experimental evidence to support SET mechanism. (C) Deuterium labeling study to identify the source of proton and the fate of carboxylic acid. (D) Frequency-dependent reaction profile for carbonyl reduction, oxidative decarboxylation, and Shono oxidation. (E) Proposed mechanism based on Figure 4A-4D. (F) Demonstration of precise redox reaction based on the understanding of rAP chemoselectivity.

\section{ASSOCIATED CONTENT}

\section{Supporting Information}

Experimental procedures, characterizations, and the detail of DFT calculation.

\section{AUTHOR INFORMATION}

\section{Corresponding Author}

*yukawama@scripps.edu

*pbaran@scripps.edu

\section{Author Contributions}


The manuscript was written through contributions of all authors. All authors have given approval to the final version of the manuscript.

\section{Notes}

YK is a consultant for IKA Works, Inc. Invention disclosure has been filed for applications of rAP into electrosynthesis.

\section{ACKNOWLEDGMENT}

Financial support for this work was provided by National Science Foundation Center for Synthetic Organic Electrochemistry (CHE-2002158), and the National Institutes of Health (grant number GM-118176). M.S. was supported by Postdoctoral Fellowship for Research Abroad (JSPS). We thank Dr. D.-H. Huang and Dr. L. Pasternack (Scripps Research) for assistance with NMR spectroscopy; Dr. J. Chen (Automated Synthesis Facility, Scripps Research) for purification of compounds and acquisition of HRMS data; Dr. A. Ramirez (BMS) for DFT calculation; M. Bird (Scripps Research) for the synthesis of oligopeptides. We would like to acknowledge the BMS biology team for their contributions running the cereblon binding assay. We also thank Dr. S. B. J. Kan and Dr. S. Gnaim for helpful advice and suggestions.

\section{REFERENCES}

Shenvi, R. A.; O'Malley, D. P.; Baran, P. S. Chemoselectivity: The mother of invention in total synthesis. Acc. Chem. Res. 2009, 42, 530-541.

M. A. Sierra; M. C. de la Torre; F. P. Cossio. More Dead Ends and Detours: En Route to Successful Total Synthesis; Wiley, 2013.

T. Yao. Comprehensive Organic Transformations: A Guide to Functional Group Preparations, R. C. Larock, Ed., 3rd ed.; Wiley, 2018.

Wuts, P. G. M. Greene's Protective Groups in Organic Synthesis, 5th ed.; Wiley, 2014.

Yan, M.; Kawamata, Y.; Baran, P. S. Synthetic organic electrochemical methods since 2000: On the verge of a renaissance. Chem. Rev. 2017, 117, 13230-13319.

Roth, H.; Romero, N.; Nicewicz, D. Experimental and Calculated Electrochemical Potentials of Common Organic Molecules for Applications to Single-Electron Redox Chemistry. Synlett 2016, 27, 714-723.

Alternating polarity commonly refers to switching electrode polarity periodically at intervals of several seconds to minutes to alleviate electrode fouling. This type of slow alternating current process is not included within the scope of this study.

Rodrigo, S.; Gunasekera, D.; Mahajan, J. P.; Luo, L. Alternating Current Electrolysis for Organic Synthesis. Current Opinion in Electrochemistry 2021, 28, 100712.

Schotten, C.; Taylor, C. J.; Bourne, R. A.; Chamberlain, T. W.; Nguyen, B. N.; Kapur, N.; Willans, C. E. Alternating polarity for enhanced electrochemical synthesis. React. Chem. Eng. 2021, 6, 147-151.

Rodrigo, S.; Um, C.; Mixdorf, J. C.; Gunasekera, D.; Nguyen, H. M.; Luo, L. Alternating current electrolysis for organic electrosynthesis: Trifluoromethylation of (hetero)arenes. Org. Lett. 2020, 22, 6719-6723. 
Bortnikov, E. O.; Semenov, S. N. Coupling of alternating current to transition-metal catalysis: Examples of nickel-catalyzed cross-coupling. J. Org. Chem. 2021, 86, 782793.

Sattler, L. E.; Otten, C. J.; Hilt, G. Alternating current electrolysis for the electrocatalytic synthesis of mixed disulfide via sulfur-sulfur bond metathesis towards dynamic disulfide libraries. Chem. Eur. J. 2020, 26, 3129-3136.

Lee, B.; Naito, H.; Nagao, M.; Hibino, T. Alternating-current electrolysis for the production of phenol from benzene. Angew. Chem. Int. Ed. 2012, 51, 6961-6965. Yan, M.; Kawamata, Y.; Baran, P. S. Synthetic Organic Electrochemistry: Calling All Engineers. Angew. Chem. Int. Ed. 2017, 57, 4149-4155.

Nouri-Nigjeh, E.; Permentier, H. P.; Bischoff, R.; Bruins, A. P. Electrochemical oxidation by square-wave potential pulses in the imitation of oxidative drug metabolism. Anal. Chem. 2011, 83, 5519-5525.

Wattanakit, C.; Yutthalekha, T.; Asssavapanumat, S.; Lapeyre, V.; Kuhn, A. Pulsed electroconversion for highly selective enantiomer synthesis. Nature Communications 2017, 8, 1-8.

Bakshi, R.; Fedkiw, P. S. Optimal Time-Varying Potential Control. J. Appl. Electrochem. 1993, 23, 715-727.

Blanco, D. E.; Lee, B.; Modestino, M. A. Optimizing organic electrosynthesis through controlled voltage dosing and artificial intelligence. Proc Natl Acad Sci U S A 2019, 116, 17683-17689.

Yamamoto, K.; Kuriyama, M.; Onomura, O. Shono-type oxidation for functionalization of N-heterocycles. Chem Rec. 2021, 10.1002/tcr.202100031. Bai, Y.; Shi, L.; Zheng, L.; Ning, S.; Che, X.; Zhang, Z.; Xiang, J. Electroselective and controlled reduction of cyclic imides to hydroxylactams and lactams. Org. Lett. 2021, 23, 2298-2302.

Hu, S.; Yuan, L.; Yan, H.; Li, Z. Design, synthesis and biological evaluation of Lenalidomide derivatives as tumor angiogenesis inhibitor. Bioorg. Med. Chem. Lett. 2017, 27, 4075-4081.

Luzzio, F. A.; Mayorov, A. V.; Ng, S. S. W.; Kruger, E. A.; Figg, W. D. Thalidomide metabolites and analogues. 3 . Synthesis and antiangiogenic activity of the teratogenic and TNF $\alpha$-modulatory thalidomide analogue 2-(2,6-dioxopiperidine-3-

yl)phthalimidine. J. Med. Chem. 2003, 46, 3793-3799.

Ding, G.; Li, C.; Shen, Y.; Lu, B.; Zhang, Z.; Xie, X. Potassium hydroxide-catalyzed chemoselective reduction of cyclic imides with hydrosilanes: Synthesis of $\omega$ hydroxylactams and lactams. Adv. Synth. Catal. 2016, 358, 1241-1250.

Park, H.; Yu, J.-Q. Palladium-catalyzed [3 + 2] cycloaddition via twofold 1,3-C( $\left(\mathrm{sp}^{3}\right)-$ $\mathrm{H}$ activation. J. Am. Chem. Soc. 2020, 142, 16552-16556.

Das, S.; Addis, D.; Knöpke, L. R.; Bentrup, U.; Junge, K.; Brückner, A.; Beller, M. Selective catalytic monoreduction of phthalimides and imidazolidine-2,4-diones. Angew. Chem. Int. Ed. 2011, 50, 9180-9184.

Yu, Y.-J.; Zhang, F.-L.; Peng, T.-Y.; Wang, C.-L.; Cheng, J.; Chen, C.; Houk, K. N.; Wang, Y.-F. Sequential C-F bond functionalizations of trifluoroacetamides and acetates via spin-center shifts. Science 2021, 371, 1232-1240.

Guo, Y.-A.; Zhao, M.; Xu, Z.; Ye, T. Total synthesis and stereochemical assignment of actinoranone. Chem. Eur. J. 2017, 23, 3572-3576. 
(28) Evans, D. A.; Bilodeau, M. T.; Somers, T. C.; Clardy, J.; Cherry, D.; Kato, Y. Enantioselective Michael reactions. Diastereoselective reactions of chlorotitanium enolates of chiral $N$-acyloxazolidinones with representative electrophilic olefins. $J$. Org. Chem. 1991, 56, 5750-5752.

(29) Petzold, G.; Fischer, E. S.; Thomä, N. H. Structural basis of lenalidomide-induced CK1 $\alpha$ degradation by the CRL4 ${ }^{\mathrm{CRBN}}$ ubiquitin ligase. Nature 2016, 532, 127-130.

(30) Wurz, R. P.; Dellamaggiore, K.; Dou, H.; Javier, N.; Lo, M.-C.; McCarter, J. D.; Mohl, D.; Sastri, C.; Lipford, J. R.; Cee, V. J. A “click chemistry platform" for the rapid synthesis of bispecific molecules for inducing protein degradation. $J$. Med. Chem. 2018, 61, 453-461.

(31) Crew, A. P.; Crews, C.; Dong, H.; Wang, J.; Qian, Y.; Siu, K.; Jin, M. Imide-based modulators of proteolysis and associated methods of use. US2016/0058872, 2016. Degorce, F.; Card, A.; Soh, S.; chemical, E. T. C. HTRF: A technology tailored for drug discovery-a review of theoretical aspects and recent applications Curr. Chem. Genomics, 2009, 3, 22-32.

Griesbeck, A. G.; Schieffer, S. Electron-Transfer Reactions of Carbonyl Compounds; Wiley, 2001, pp 457-493.

Ashby, E. C.; Laemmle, J. T. Stereochemistry of Organometallic Compound Addition to Ketones. Chem. Rev. 1975, 75, 521-546.

Bunnett, J. F. Aromatic substitution by the $\mathrm{S}_{\mathrm{RN}} 1$ mechanism. Acc. Chem. Res. 1978, 11, 413-420.

Leech, M. C.; Lam, K. Electrosynthesis using carboxylic acid derivatives: New tricks for old reactions. Acc. Chem. Res. 2020, 53, 121-134.

Alfonso-Súarez, P.; Kolliopoulos, A. V.; Smith, J. P.; Banks, C. E.; Jones, A. M. An experimentalist's guide to electrosynthesis: The Shono oxidation. Tetrahedron Lett. 2015, 56, 6863-6867.

Wang, F.; Stahl, S. S. Electrochemical oxidation of organic molecules at lower overpotential: Accessing broader functional group compatibility with electron-proton transfer mediators. Acc. Chem. Res. 2020, 53, 561-574. 